\title{
Abiraterone in the treatment of metastatic castration-resistant prostate cancer
}

This article was published in the following Dove Press journal:

Cancer Management and Research

28 January 2014

Number of times this article has been viewed

\section{Elahe A Mostaghel}

Division of Clinical Research, Fred Hutchinson Cancer Research Center, Seattle, WA, USA
Correspondence: Elahe A Mostaghel Fred Hutchinson Cancer Research Center, Lab D4-345, Office D5-104,

I 100 Fairview Ave N, MS D5- 100

Seattle,WA 98109, USA

Tel +l 2066673506

Fax + I 2066675456

Email emostagh@fhcrc.org
Abstract: Androgen deprivation therapy remains the single most effective treatment for the initial therapy of advanced prostate cancer, but is uniformly marked by progression to castrationresistant prostate cancer (CRPC). Residual tumor androgens and androgen axis activation are now recognized to play a prominent role in mediating CRPC progression. Despite suppression of circulating testosterone to castrate levels, castration does not eliminate androgens from the prostate tumor microenvironment and residual androgen levels are well within the range capable of activating the androgen receptor (AR) and AR-mediated gene expression. Accordingly, therapeutic strategies that more effectively target production of intratumoral androgens are necessary. The introduction of abiraterone, a potent suppressor of cytochrome P450 $17 \alpha$-hydroxysteroid dehydrogenase-mediated androgen production, has heralded a new era in the hormonal treatment of men with metastatic CRPC. Herein, the androgen and ARmediated mechanisms that contribute to CRPC progression and establish cytochrome P450 $17 \alpha$-hydroxysteroid dehydrogenase as a critical therapeutic target are briefly reviewed. The mechanism of action and pharmacokinetics of abiraterone are reviewed and its recently described activity against AR and 3- $\beta$-hydroxysteroid dehydrogenase is discussed. The Phase I and II data initially demonstrating the efficacy of abiraterone and Phase III data supporting its approval for patients with metastatic CRPC are reviewed. The safety and tolerability of abiraterone, including the incidence and management of side effects and potential drug interactions, are discussed. The current place of abiraterone in CRPC therapy is reviewed and early evidence regarding cross-resistance of abiraterone with taxane therapy, mechanisms of resistance to abiraterone, and observations of an abiraterone withdrawal response are presented. Future directions in the use of abiraterone, including optimal dosing strategies, the role of abiraterone in earlier disease settings, including castration sensitive, biochemically recurrent, or localized disease, and the rationale for combinatorial treatment strategies of abiraterone with enzalutamide and other targeted agents are also discussed.

Keywords: castration-resistant, abiraterone, CYP17A, androgen, intracrine

\section{Introduction to castration-resistant prostate cancer (CRPC)}

The primary treatment modality for patients with metastatic prostate cancer is androgen deprivation therapy (ADT). However, treatment is uniformly marked by progression to CRPC over a period of about 18 months, with an ensuing median survival of 1-2 years. Importantly, it is now clear that "androgen independent" or "hormone refractory" tumors remain sensitive to hormonal activation, and that despite suppression of circulating testosterone $(\mathrm{T})$, residual tumor androgens and androgen axis activation play a prominent role in mediating CRPC progression. ${ }^{1}$ Numerous molecular features 
have been shown to contribute to AR signaling in CRPC and demonstrate that ongoing AR activation may occur via both ligand-dependent and ligand-independent mechanisms. As a consequence, the efficiency of AR activation at low or absent ligand levels can be enhanced and AR ligand specificity can be broadened, potentiating the persistent activation of AR signaling in CRPC tumors.

\section{Residual tumor androgens in CRPC}

Castration does not eliminate androgens from the prostate tumor microenvironment and residual androgen levels are well within the range capable of activating the AR and AR-mediated gene expression, ${ }^{2-5}$ strongly suggesting that intratumoral androgens are clinically relevant in driving castration-resistant tumors. While the efficacy of ADT is based on achieving castrate levels of serum $\mathrm{T}$ (defined as $<20 \mathrm{ng} / \mathrm{dL}$ ), measurement of prostatic tissue androgen levels in locally recurrent and metastatic CRPC has consistently demonstrated the presence of residual tumor androgens. In advanced prostate cancer, Mohler et al found that prostatic $\mathrm{T}$ levels in castrate patients with locally recurrent tumors were equivalent to those of benign prostatic hyperplasia patients and that intratumoral dihydrotestosterone (DHT) levels were only reduced $80 \%$ (to $\sim 0.4 \mathrm{ng} / \mathrm{g}$ ). ${ }^{3}$ In another study, T levels in metastatic tumors obtained via rapid autopsy from men with CRPC were found to be approximately three-fold higher than $\mathrm{T}$ levels within primary prostate tumors from untreated (eugonadal) patients (T $0.74 \mathrm{ng} / \mathrm{g}$; DHT $0.25 \mathrm{ng} / \mathrm{g}$ ). ${ }^{6}$

Data derived from in vitro and in vivo studies have determined that tissue DHT levels of $0.5-1.0 \mathrm{ng} / \mathrm{g}$ (the range observed in prostatic tissue of castrated patients) are sufficient to activate the AR, stimulate expression of AR-regulated genes, and promote androgen-mediated tumor growth. ${ }^{3,7-10}$ Moreover, residual tissue androgens participate in nearly every mechanism by which AR-mediated signaling leads to the development of castration-resistant disease, including AR overexpression, AR mutations that alter ligand binding, and alterations in AR coregulators, all of which result in hypersensitization of AR to activation by low levels of residual androgens. ${ }^{11}$ The maintenance of intratumoral androgens can be accounted for, in part, by intratumoral or intracrine biosynthesis of steroid hormones, either via the uptake and conversion of adrenal androgens (as initially put forward by Labrie et al), ${ }^{12}$ or potentially via de novo steroidogenesis. ${ }^{6,13-18}$

\section{$A R$ alterations in CRPC}

AR overexpression is a well-recognized feature of CRPC and believed to be a critical driver of CRPC progression. . $^{31,19-27}$
Potential mechanisms responsible for increased AR expression include amplification of the AR locus itself, increased transcription rates, or stabilization of the messenger RNA or protein. AR overexpression not only mediates sensitivity to low ligand concentrations, but has been demonstrated to convert anti-androgens such as bicalutamide and flutamide from antagonists to agonists via changes in composition of coactivators recruited to the AR promoter. ${ }^{28,29}$

Several well-characterized AR mutations can result in promiscuous binding and activation of the AR by weak adrenal androgens and other steroid hormones, including dehydroepiandrosterone (DHEA), progesterone, estrogens, and cortisol. ${ }^{30-35}$ Other mutations convert AR antagonists (flutamide and bicalutamide) into potent agonists. ${ }^{36}$ Notably, in vitro selection with enzalutamide has revealed a new mutation (F876L) which mediates conversion of enzalutamide to an AR agonist while maintaining sensitivity to bicalutamide. ${ }^{37,38}$ This mutation also confers resistance to the second-generation AR antagonist ARN509, and has been detected in circulating tumor DNA from ARN509-treated patients. ${ }^{39}$ While the frequency of AR mutation in CRPC tumors treated with luteinizing hormone (LH)-releasing hormone agonist and first-generation AR antagonists is low $(8 \%-25 \%),{ }^{33}$ the frequency of these mutations may become more frequent with the advent of potent antagonists of AR signaling.

Constitutively active truncated AR splice variants have recently been recognized as a potential mechanism of CRPC progression. The expression of certain variants (eg, AR-V7, which can be detected in hormone-naïve prostate cancer) has been associated with a shorter time to disease recurrence following radical prostatectomy. ${ }^{40,41}$ High levels of ARV7 and ARV567 were associated with shorter survival in patients with CRPC and bone metastases, consistent with a role in tumor progression. ${ }^{42,43}$ Although each variant displays a slightly different structure, they each lack portions of the carboxy-terminal ligand binding domain, resulting in a constitutively active AR. ${ }^{44}$

\section{Clinical evidence of AR axis signaling in CRPC}

The clinical importance of ongoing AR pathway activity in CRPC progression is reflected in the rising serum prostatespecific antigen (PSA) levels in patients with CRPC, and is confirmed by clinical responses to treatment strategies that target residual AR axis activity. These include historical responses to adrenalectomy and/or hypophysectomy; ${ }^{45,46}$ the limited but consistent $\sim 5 \%$ overall survival (OS) benefit seen 
in meta-analyses of combined androgen blockade trials; $;{ }^{47-49}$ the observation that nearly $30 \%$ of recurrent prostate tumors demonstrate at least transient clinical responses to secondary or tertiary hormonal manipulation; ${ }^{50}$ and most recently, the striking clinical response observed with the novel AR axis inhibitors abiraterone and MDV3100. ${ }^{51,52}$

\section{Mechanism of action and pharmacology of abiraterone}

Emerging data suggest residual intratumoral androgens are produced via the uptake and conversion of adrenal androgens, and potentially via de novo synthesis from cholesterol or progesterone precursors within the tumor. ${ }^{6,13-18}$ Accordingly, therapeutic strategies that more effectively target production of intratumoral androgens are necessary. Abiraterone is the first to enter clinical practice in a series of novel agents designed to potently target adrenal and tumor androgen production.

\section{Cytochrome P450 I7 $\alpha$-hydroxysteroid dehydrogenase (CYPI7A) as a therapeutic target in CRPC}

The critical enzyme required for androgen synthesis from cholesterol is CYP17A. Adrenal expression of this enzyme accounts for production of circulating adrenal androgens, including DHEA (which primarily circulates in its sulfated form, DHEA-S) and androstenedione (AED), and a number of studies have demonstrated expression of CYP17A in castration-resistant prostate tumors. ${ }^{6,53}$ Given its central role in the production of either adrenal or tumor-derived extragonadal androgen synthesis, CYP17A has emerged as a primary target of novel therapeutics.

CYP17A is a single enzyme that catalyzes the sequential hydroxylase (required for cortisol synthesis) and lyase (required for adrenal androgen synthesis) steps that are required for conversion of $\mathrm{C} 21$ pregnenolone and progesterone precursors to the $\mathrm{C} 19$ adrenal androgens, DHEA and AED. While ketoconazole (a weak inhibitor of CYP11 $\alpha$ hydroxylase and CYP17A) has been utilized for suppression of residual adrenal androgens in men with CRPC, its limited efficacy and treatment-related side effects prompted the development of more potent CYP17A inhibitors. ${ }^{54}$

Abiraterone acetate is an orally administered, rationally designed small molecule derived from the structure of pregnenolone. It irreversibly inhibits both the hydroxylase and lyase activity of CYP17A with approximately 10-30fold greater potency than ketoconazole. ${ }^{55}$ Because adrenal inhibition of CYP17A results in blockade of glucocorticoid as well as adrenal androgen synthesis, abiraterone is coadministered with prednisone to ameliorate the secondary rise in adrenocorticotropic hormone (ACTH) that can lead to excess mineralocorticoid synthesis (Figure 1, discussed further below). ${ }^{56}$

\section{Unintended activity of abiraterone against other $A R$ pathway targets}

Abiraterone was designed as a selective, irreversible inhibitor of CYP17A. However, most likely due to its steroidal structure, abiraterone has been found to inhibit other AR pathway targets including AR itself, as well as 3 $\beta$-hydroxysteroid dehydrogenase, another key enzyme required for androgen synthesis.

While not as potent as the first-generation nonsteroidal AR inhibitors (eg, bicalutamide), abiraterone demonstrated measurable AR antagonism at $1-10 \mu \mathrm{mol} / \mathrm{L}$, a clinically achievable concentration. ${ }^{57}$ Pharmacokinetic studies (discussed below) have found plasma abiraterone levels to be $1.2-5 \mu \mathrm{mol} / \mathrm{L}$ following a $1,000 \mathrm{mg}$ dose in fasting patients. Galeterone, another novel CYP17A antagonist, has been demonstrated to have anti-AR activity as well, and actually has increased potency compared to abiraterone in this regard.$^{58}$ Interestingly, an early study demonstrated that ketoconazole can also inhibit the AR, although not at clinically achievable doses. ${ }^{59}$

With regard to its activity against $3 \beta$-hydroxysteroid dehydrogenase type I, abiraterone was shown to inhibit two key reactions mediated by this enzyme. Abiraterone, again at clinically achievable concentrations of $2.1-8.8 \mu \mathrm{mol} / \mathrm{L}$, inhibited the conversion of DHEA to AED, and of $5 \alpha$-androstanediol to $\mathrm{T}$, with concomitant suppression of AR-regulated gene expression. ${ }^{60}$ These data suggest that even though maximal inhibition of CYP17A is achieved at the currently approved 1,000 $\mathrm{mg}$ dose, dose escalation may increase the efficacy of abiraterone by taking advantage of its ability to inhibit multiple AR pathway targets. Clinical trials evaluating this hypothesis are ongoing.

\section{Pharmacokinetics of abiraterone}

Abiraterone is administered as the pro-drug abiraterone acetate which has improved oral bioavailability and shows near complete conversion to abiraterone in the blood. In preclinical toxicology studies, abiraterone reduced the weights of androgen dependent organs such as the prostate and had minimal side effects in other organs. ${ }^{61}$ First-in-man studies demonstrated the ability of abiraterone to reduce 


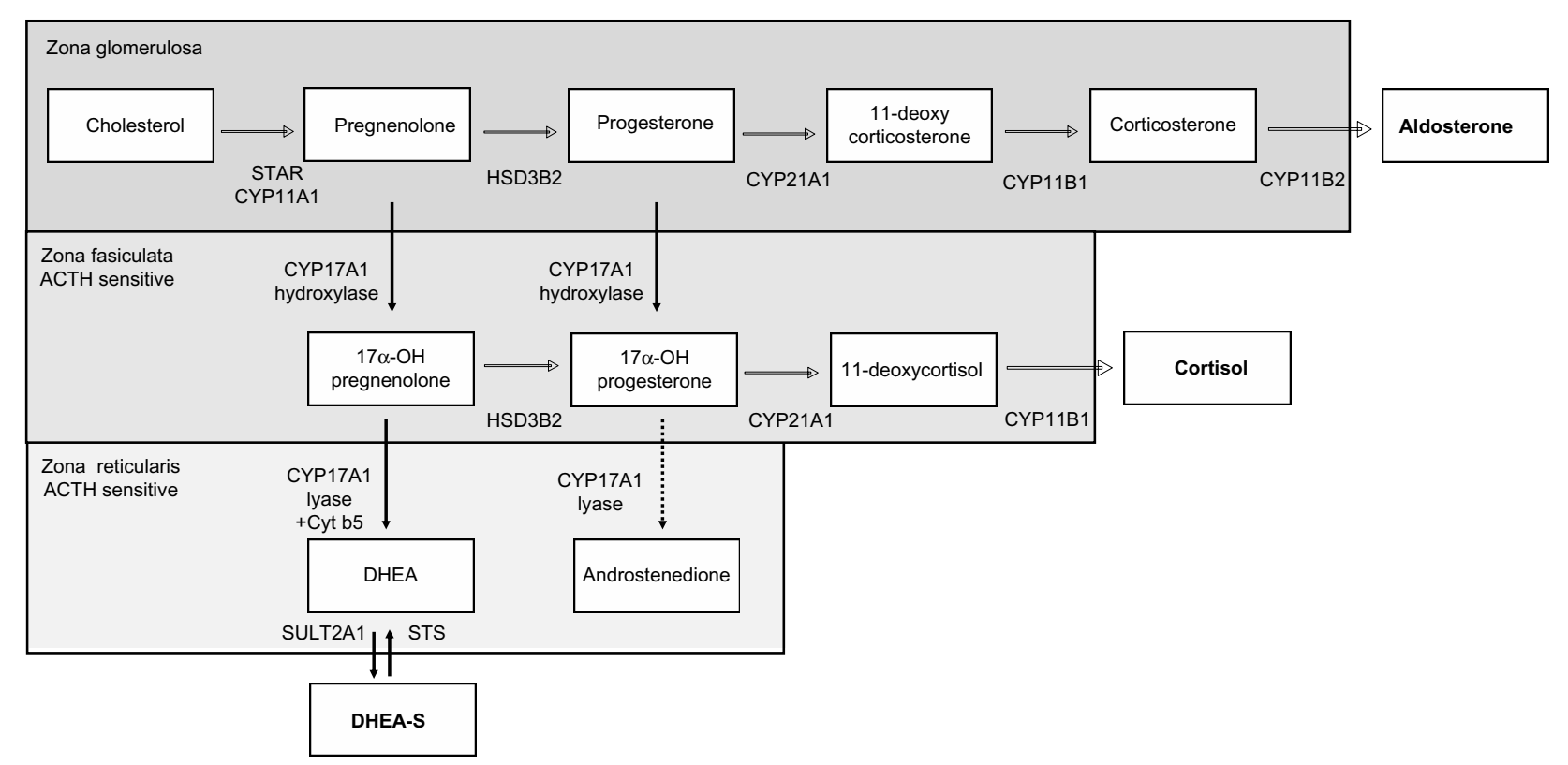

Figure I Steroid hormone pathways in zones of the adrenal gland. Steroid synthesis in the adrenal gland occurs in three zones, each with a specific complement of enzymes that determine the steroids produced. The zona glomerulosa contains the enzymes necessary to produce aldosterone. The zona fasciculata and reticularis additionally express CYPI7A. The hydroxylase activity of CYPI7A is active in the zona fasciculata resulting in the production of cortisol. However, the lyase activity of CYPI7A requires the cytochrome b5 coregulator which is only present in the zona reticularis. This drives efficient production of DHEA which is then sulfated to DHEA-S. I7 $\alpha-O H$ progesterone is a relatively poor substrate for CYPI7A lyase (dotted arrow), and thus androstenedione is formed at lower levels. The zona fasciculata and zona reticularis are sensitive to the ACTH feedback stimulation that occurs when cortisol production is suppressed by inhibition of CYPI7A.

Abbreviations: I $7 \alpha-\mathrm{OH}$, I $7 \alpha$-hydroxy; ACTH, adrenocorticotropic hormone; CYPI IA, cytochrome P450 II $\alpha$-hydroxylase; CYPI IB, cytochrome P450 I I $\beta$-hydroxylase; CYPI7A, I7 $\alpha$-hydroxylase/I7,20 lyase/I7,20 desmolase; CYP2IA, cytochrome P450 2I $\alpha$-hydroxylase; Cyt b5, cytochrome b5; DHEA, dehydroepiandrosterone; DHEA-S, dehydroepiandrosterone-sulfate; HSD3B, 3- $\beta$-hydroxysteroid dehydrogenase; STAR, steroidogenic acute regulatory protein.

serum $\mathrm{T}$ levels in both castrate and noncastrate men. In castrate men dose escalation to $500 \mathrm{mg}$ was required to achieve the target effect, a $75 \%$ decrease in $\mathrm{T}$ levels. The duration of suppression following a single dose was variable, ranging from 2-5 days. In noncastrate men, repeat dosing at $\geq 800 \mathrm{mg}$ daily was required to maintain $\mathrm{T}$ suppression, and was accompanied by a marked rise in LH that may have prevented sustained T suppression. ${ }^{62}$

Two Phase I trials in chemotherapy-naïve men with metastatic CRPC were performed to determine the recommended dose for Phase II testing. No dose-limiting toxicities were observed at doses up to $2,000 \mathrm{mg} /$ daily, but $1,000 \mathrm{mg}$ was chosen for expansion as a plateau in pharmacodynamic effect was observed at doses $>750 \mathrm{mg}$. Substantial variability in drug absorption was observed, with up to nine-fold differences in serum pharmacokinetic parameters within the $1,000 \mathrm{mg}$ cohort. Maximal drug concentrations were achieved within 2-4 hours, with a terminal half-life of approximately 12 hours..$^{63,64}$

\section{Impact of food on abiraterone exposure}

Notably, drug exposure was five to seven-fold higher when abiraterone was administered with a low-fat meal (7\% fat, 300 calories) and over ten-fold higher with a high-fat meal
( $57 \%$ fat, 825 calories) as compared to the fasted state. To minimize the variability in absorption, abiraterone is labeled for administration as $1,000 \mathrm{mg}$ (four $250 \mathrm{mg}$ tablets) daily on an empty stomach, defined as 1 hour before or 2 hours after a meal. ${ }^{65}$ Clinical trials evaluating abiraterone in the fed and fasted state are ongoing (NCT01424930), ${ }^{66}$ as are trials evaluating lower doses of abiraterone taken with food (NCT01543776). ${ }^{67}$ If borne out, these approaches could decrease drug costs, as well as decrease the potential risk of drug interactions (see below) if a patient accidentally takes the agent with food. ${ }^{65,68}$

In a dedicated renal impairment trial, renal dysfunction did not impact pharmacokinetic profiles and no dose adjustment is necessary for renal impairment. ${ }^{69}$ Abiraterone is bound to plasma proteins including albumin and is primarily excreted in the feces. ${ }^{70}$

\section{Efficacy studies Phase I and II data}

Phase I and II studies demonstrated that abiraterone suppresses serum androgen levels, and achieves PSA and clinical responses in chemotherapy-naïve and docetaxeltreated CRPC patients. No dose-limiting toxicities were associated with abiraterone, and anti-tumor activity and 
PSA responses were observed at all dose levels. While the highest tolerated dose was $2,000 \mathrm{mg} /$ day, all Phase II and III studies have used the $1,000 \mathrm{mg} /$ day dose, as the impact of therapy on steroids upstream of CYP17A plateaued at doses $>750 \mathrm{mg} /$ day. $^{63}$

In eugonadal men, abiraterone transiently suppresses $\mathrm{T}$ levels by $>50 \%$ (but a corresponding rise in LH levels overcomes inhibition of gonadal androgen synthesis), while in castrate men, abiraterone further suppresses castrate serum $\mathrm{T}$ levels by $\geq 75 \%$. ${ }^{62}$ In general, abiraterone suppresses serum DHEA levels by approximately $75 \%$ and DHEA-S, AED, and T to essentially undetectable. ${ }^{63,64}$ As observed in studies of ketoconazole, higher baseline levels of DHEA-S, DHEA, and AED were present in patients achieving $>50 \%$ PSA declines. In contrast to progression on ketoconazole, increases in T, AED, or DHEA levels were not observed on progression with abiraterone..$^{71,72}$

In men with chemotherapy- and ketoconazole-naïve metastatic CRPC, a Phase I/II single-agent study of abiraterone demonstrated durable PSA declines $>50 \%$ in $67 \%$ of patients, with partial radiographic responses in $37.5 \%$ and a median time to progression (TTP) of 32 weeks. ${ }^{72}$ Based on preclinical data that progesterone precursors upstream of CYP17A can activate certain AR mutations, this study also evaluated the addition of corticosteroids at progression and showed that $33 \%$ of patients $(10 / 30)$ had secondary PSA responses following the addition of dexamethasone $0.5 \mathrm{mg} /$ day. ${ }^{72}$ A Phase II study of abiraterone combined with prednisone in this population demonstrated PSA declines $>50 \%$ in $79 \%$ of patients and a median TTP of 65 weeks. This study also found that over $48 \%(11 / 23)$ evaluable patients had a transient bone scan flare at 3 months that subsequently showed with improvement or stability. ${ }^{73}$ In a second Phase I single-agent study of pre-chemotherapy patients in which $58 \%$ were ketoconazole pretreated, PSA declines $>50 \%$ were noted in $55 \%$ of patients at 12 weeks. ${ }^{64}$

In post-docetaxel-treated CRPC patients, a Phase II singleagent study of abiraterone demonstrated PSA declines $>50 \%$ in $51 \%$ of patients (only $17 \%$ had received prior ketoconazole), with partial radiographic responses in $27 \%$ and a median TTP of 24 weeks. ${ }^{74}$ In a Phase II study of abiraterone combined with prednisone, in which $47 \%$ of patients were ketoconazole pretreated, PSA declines $>50 \%$ were observed in $36 \%$ of patients with a median TTP of 24 weeks. $^{75}$

\section{Efficacy in ketoconazole-treated patients}

Importantly, while ketoconazole-treated patients were specifically excluded in the subsequent Phase III studies, the Phase I/II studies demonstrated that abiraterone has activity in these patients as well. In the pre-chemotherapy Phase I study in which $58 \%$ of men (19 of 33) were ketoconazole pretreated, PSA responses $>50 \%$ were observed in $64 \%$ of ketoconazole-naïve patients and $47 \%$ of ketoconazolepretreated patients. ${ }^{64}$ In the post-docetaxel study in which $47 \%$ of patients ( 27 of 58 ) had received prior ketoconazole, PSA declines $>50 \%$ occurred in $45 \%$ of ketoconazole-naïve patients and $26 \%$ of ketoconazole-treated patients with a median TTP of 28 and 14 weeks, respectively. ${ }^{75}$ These findings demonstrate that abiraterone maintains a reasonable degree of clinical efficacy in CRPC patients previously treated with docetaxel and/or ketoconazole.

\section{Phase III data}

Findings from the Phase I and II studies have been subsequently confirmed in Phase III studies in chemotherapynaïve (COU-AA-302) and post-docetaxel-treated men (COU-AA-301), resulting in US Food and Drug Administration approval of abiraterone for men with metastatic CRPC either before or after treatment with chemotherapy.

\section{COU-AA-30I}

In the post-chemotherapy setting, 1,195 men with metastatic CRPC progressing after docetaxel were randomized in a 2:1 ratio to abiraterone/prednisone $(n=797)$ or placebo/prednisone $(n=398)$ with a primary endpoint of OS. The median PSA was $\sim 130 \mathrm{ng} / \mathrm{dL}, 90 \%$ of patients had an Eastern Cooperative Oncology Group performance status (PS) of zero to one, the median age was 70 years, and $28 \%$ were $\geq 75$ years. Bone, lymph node, and visceral metastases were present in approximately $90 \%, 40 \%$, and $10 \%$ of patients, respectively, and $30 \%$ of patients had received more than one prior chemotherapy regimen. Treatment was continued until clinical or radiographic evidence of progression.

At a median follow-up of 12.8 months, the first interim analysis demonstrated an OS benefit for men receiving abiraterone (14.8 months versus 10.9 months for placebo; hazard ratio [HR] $0.646 ; P<0.0001$ ), representing a $35 \%$ reduction in risk of death and prompting the independent data monitoring committee to recommend that the study be unblinded and men on the placebo arm be offered abiraterone. ${ }^{51}$ An updated analysis at a median survival of 20.2 months demonstrated a median OS of 15.8 months for abiraterone versus 11.2 months for prednisone (HR 0.74; $P<0.0001$ ), extending the OS benefit to 4.6 months.

All secondary endpoints were statistically significant in favor of abiraterone, including median time to PSA 
progression (8.5 months versus 6.6 months), median radiologic progression-free survival (rPFS; 5.6 months versus 3.6 months), and proportion of patients with $>50 \%$ PSA response $(29.5 \%$ versus $5.5 \%$ ). The impact of abiraterone on OS was observed across all subgroups, including patients who had received one (15.4 versus 11.5 months) or two prior chemotherapy regimens (14.0 versus 10.3 months). Notably, patients with a PS of two had worse outcomes, with a median survival of 7.3 months on abiraterone compared to 15.3 months for those with PS of zero to one receiving abiraterone. ${ }^{76}$

While visceral disease was associated with a poorer prognosis, an exploratory analysis reported in abstract form found the absolute benefit in OS from abiraterone to be similar in those with and without visceral disease (8.3-12.9 months in those with visceral disease and 12.3-17.3 months in those without). Analysis of patients by site of disease showed worse outcomes in those with hepatic versus pulmonary visceral metastases, but still a benefit favoring abiraterone over placebo (median OS 4.0-7.3 months for liver metastases and 7.9-13.9 months for pulmonary disease). ${ }^{77}$

Exploratory analyses of COU-AA-301 evaluating the impact of abiraterone on fatigue, pain control, and skeletalrelated events suggest abiraterone has efficacy in all these settings. In patients with clinically significant fatigue at baseline, abiraterone significantly increased the number of patients reporting an improvement in fatigue intensity $(58.1 \%$ versus $40.3 \%$; $P=0.0001$ ) as well as the time to fatigue palliation (median 59 days versus 194 days; $P=0.0155) .{ }^{78}$ In patients with clinically significant pain at baseline, abiraterone significantly increased the number of patients reporting palliation of pain ( $45 \%$ versus $28.8 \% ; P=0.0005$ ), as well as faster palliation (median time to palliation 5.6 months versus 13.7 months; $P=0.0018$ ). Median time to occurrence of first skeletal-related event (defined as pathologic fracture, spinal cord compression, or palliative surgery or radiation to bone) was also significantly longer in abiraterone treated patients (25 months versus 20.3 months; $P=0.0001$ ). ${ }^{79}$

\section{COU-AA-302}

In the pre-chemotherapy setting, 1,088 men with asymptomatic or minimally symptomatic bone and lymph node (but not visceral) metastatic CRPC were randomized 1:1 to abiraterone/prednisone $(n=546)$ or placebo/prednisone $(n=542)$, with co-primary endpoints of rPFS and OS. The median PSA was $\sim 40 \mathrm{ng} / \mathrm{dL}$, about $30 \%$ of men were $\geq 75$ years, and approximately $50 \%$ had bone-only metastatic disease.

At a median follow-up of 22.2 months, a statistically significant doubling in rPFS from 8.3 months in the placebo arm to 16.5 months was observed in men receiving abiraterone (HR $0.53 ; P<0.001$ ), accompanied by a trend for increased OS from 27.3 months in the placebo arm to not reached in the abiraterone group (HR $0.75 ; P=0.01$ which did not meet the prespecified $P$-value of 0.001 ), again prompting the independent data monitoring committee to recommend that the study be unblinded and men on the placebo arm be offered abiraterone. ${ }^{80}$ An updated analysis of OS at a median survival of 27.1 months again trended toward favoring abiraterone at 30.1 months in the placebo arm versus 35.3 months in the abiraterone arm (HR 0.79; $P=0.015) .{ }^{81}$

All secondary endpoints were statistically significant in favor of abiraterone, including median time to opiate use (not reached versus 23.7 months), time to initiation of chemotherapy (25.2 months versus 16.8 months), time to PS decline (12.3 months versus 10.9 months), time to PSA progression (11.1 months versus 5.6 months), and proportion of patients with $>50 \%$ PSA response (62\% versus $24 \%$ ). The impact of abiraterone on rPFS was observed across all subgroups. ${ }^{80}$ This study did not include patients with visceral disease or moderate to severe pain; however, the exploratory analyses of these subpopulations in the post-chemotherapy setting discussed above suggest these patients are likely to benefit as well.

\section{Safety and tolerability}

Abiraterone is generally well tolerated, with $13 \%$ and $19 \%$ of abiraterone-treated patients in COU-AA-301 and COU-AA-302, respectively, discontinuing therapy for adverse effects versus $18 \%$ and $23 \%$ of placebo-treated patients. The most common adverse events in both groups were fatigue, back pain, nausea, constipation, bone pain, and arthralgia, all in the range of $25 \%-30 \%$. The incidence of urinary tract infection was statistically higher in abirateronetreated patients $(12 \%$ versus $7 \%$ in placebo; $P=0.02)$.

\section{Mineralocorticoid and electrolyte effects}

Inhibition of CYP17A by abiraterone increases mineralocorticoid precursors upstream of CYP17A and decreases glucocorticoids downstream of CYP17A (Figure 1). The latter results in a concomitant elevation of $\mathrm{ACTH}$, which further drives mineralocorticoid production. Phase I and II trials demonstrated that symptoms of mineralocorticoid excess occur in $50 \%-80 \%$ of patients treated with single-agent abiraterone. ${ }^{56}$ Mineralocorticoid-related symptoms in the Phase III studies were markedly attenuated by inclusion of prednisone $5 \mathrm{mg}$ twice daily, and were generally of Grade I or II in magnitude, including fluid retention ( $-33 \%$ versus $22 \%-24 \%$ in placebo), 
hypertension ( $10 \%$ versus $8 \%$ in placebo), and hypokalemia ( $\sim 18 \%$ versus $9 \%$ in placebo).

Dexamethasone, which lacks mineralocorticoid effects and has a longer duration of action, may theoretically be preferable to prednisone and can be used at a dose of $0.5 \mathrm{mg}$ daily. ${ }^{82}$ However, a rare incidence $(2 / 100)$ of orthostatic hypotension was reported following addition of dexamethasone to single-agent abiraterone, possibly due its lack of mineralocorticoid activity in the setting of a rapid decline in circulating mineralocorticoids. ${ }^{63,64}$

Of note, other CYP17A inhibitors such as orteronel and galeterone demonstrate more selective targeting of the lyase but not hydroxylase activity of CYP17A. These agents may be associated with less inhibition of cortisol synthesis and less ACTH/feedback-driven symptoms of mineralocorticoid excess and are being evaluated for use both with and without corticosteroids.

Careful attention should be paid to hypertension and hypokalemia, which should be corrected before and during therapy with abiraterone. Patients should be monitored for hypertension, hypokalemia, and fluid retention at least once a month. Spironolactone is avoided in patients who develop mineralocorticoid-related side effects due to its mixed AR agonist/antagonist activity. Instead, eplerenone, a secondgeneration mineralocorticoid receptor antagonist in doses of 50-200 mg/day (in divided doses twice daily) can be used in combination with a salt-restricted diet. ${ }^{83}$

While eplerenone does not bind the wild-type AR, it can activate certain AR mutations; however, further data is needed regarding the incidence and clinical significance of these mutations. Alternatively, potassium-sparing epithelial sodium channel antagonists such as amiloride and triamterene (in combination with hydrochlorothiazide if hypertension is significant) can be used in place of or added to eplerenone if necessary. ${ }^{82,83}$ In rare instances, additional anti-hypertensive agents may be necessary in patients already receiving prednisone, eplerenone, and diuretics.

\section{Hepatotoxicity}

Grade III or IV hepatic transaminase abnormalities (five times the upper limit of normal [ULN]) occurred in approximately $4 \%$ of patients in the Phase III studies, usually within the first 3 months of starting treatment, and more commonly in men whose baseline alanine transaminase or aspartate transaminase were elevated. Serum transaminases should be measured at baseline. Transaminases in patients with normal levels should be checked every 2 weeks for the first 3 months of therapy, and then monthly. No dose adjustment is necessary for mild hepatic impairment. For moderate hepatic impairment (Child-Pugh Class B) abiraterone should be started at $250 \mathrm{mg}$ daily, and transaminases should be checked weekly for the first month, then every 2 weeks for the following 2 months, and then monthly.

If aspartate transaminase or alanine transaminase rise above five times the ULN or bilirubin rises above three times the ULN, abiraterone should be held. It should be discontinued if the patient had moderate hepatic impairment at baseline, but in patients with normal hepatic function at baseline it can be restarted at $750 \mathrm{mg}$ daily when liver function tests decline to less than 2.5 times the ULN and total bilirubin is less than 1.5 times the ULN. If hepatotoxicity recurs, a further dose reduction to $500 \mathrm{mg}$ can be attempted (once levels have fallen below the thresholds given above), but recurrence of hepatotoxicity at the $500 \mathrm{mg}$ dose requires discontinuation of the drug.

\section{Cardiotoxicity}

The overall incidence of adverse cardiac effects was not statistically increased by abiraterone in COU-001 (13\% versus $11 \%$ in placebo), although the frequency of cardiac failure was higher in the abiraterone group (2.1\% versus $0.7 \%$ in placebo). The most frequently reported cardiac events were Grade I and II tachycardia and Grade III or lower atrial fibrillation. A retrospective study of 51 metastatic CRPC patients with at least one cardiac comorbidity and/or controlled risk factor including hypertension (41\%), hyperglycemia (30\%), dyslipidemia $(18 \%)$, cardiac ischemia $(12 \%)$, stroke $(9 \%)$, or arrhythmias $(6 \%)$ reported no cardiac events or variation in left ventricular ejection fraction over 6-12 months of follow-up. ${ }^{84}$ However, as patients with left ventricular ejection fraction $<50 \%$ were excluded from the Phase III studies, pretreatment assessment and optimization of cardiac status with electrocardiogram and echocardiography may warrant consideration in elderly patients with reduced cardiac function. A significant effect of abiraterone on the QT/QTc interval in patients with CRPC was not observed. ${ }^{85}$

\section{Potential drug interactions}

Abiraterone is a strong inhibitor of several microsomal drug metabolizing enzymes, including CYP1A2 and CYP2D6. ${ }^{86}$ Abiraterone increased systemic exposure of dextromethorphan (metabolized by CYP2D6) approximately two to three-fold, while the pharmacokinetics of theophylline (metabolized by CYP1A2) were unaffected. This suggests caution may be warranted when abiraterone is coadministered with known CYP2D6 substrates (including $\beta$-blockers, 
serotonin reuptake inhibitors, anti-arrhythmics, and neuroleptics, as well as codeine, tramadol, and - of relevance to urologic patients - tolterodine). ${ }^{87}$

Conversely, abiraterone is a substrate of CYP3A4. Interestingly, enzalutamide is a strong inducer of CYP3A4, while ketoconazole and bicalutamide are inhibitors of CYP3A4. Clinical trials evaluating the impact of rifampin (another strong inducer of CYP3A4) on abiraterone exposure (NCT01655147), ${ }^{88}$ as well as the impact of ketoconazole on prolonging abiraterone exposure (NCT01588782) ${ }^{89}$ have been completed but not yet reported. This suggests that the combination of bicalutamide and abiraterone merits clinical evaluation.

\section{Current place of abiraterone in CRPC therapy}

A number of clinical trials evaluating the sequence and combination of abiraterone with immunotherapy, chemotherapy, enzalutamide, and other novel agents are underway in men with metastatic CRPC. While the optimal place of abiraterone in prostate cancer therapy remains to be determined, current treatment decisions can be guided in part by Phase III data already available.

In men with asymptomatic or minimally symptomatic metastatic CRPC, abiraterone is an attractive first-line option given its ease of administration and relatively low toxicity profile. Similarly, the combination of abiraterone and sipuleucel $\mathrm{T}$ would likely be a well-tolerated regimen in this setting and is currently under clinical investigation (NCT01487863). ${ }^{90}$

The efficacy of abiraterone in men with symptomatic disease prior to chemotherapy has not been specifically demonstrated due to exclusion of these patients from the Phase III trial. The pace of disease may be the best guide to therapy in this setting. Patients with high Gleason scores, poor response to initial ADT, rapidly progressive disease, or poorly controlled symptoms may derive greater benefit from immediate chemotherapy, while a trial of abiraterone may be reasonable in patients with less extensive or more slowly progressing disease. ${ }^{91}$

In the post-chemotherapy setting, both abiraterone and enzalutamide are supported by Phase III data demonstrating an OS benefit, but the optimal approach to sequencing them is unknown. Retrospective evaluations of patients receiving abiraterone after enzalutamide or vice versa have shown modest response rates with a median TTP of 3-4 months. ${ }^{92-94}$ Until biomarkers to stratify patients or clinical trial data to support combination or sequencing strategies are available, the sequencing of abiraterone and enzalutamide is likely to be dictated by insurance and regulatory approvals. From a practical perspective, enzalutamide avoids the need for prednisone, although this may become less important if studies show abiraterone can be given with lower doses of prednisone (NCT01867710). ${ }^{95}$

\section{Cross-resistance with taxanes}

An emerging consideration is whether therapy with abiraterone (or enzalutamide) may influence the efficacy of subsequent chemotherapy. Taxanes have been demonstrated to mediate some of their anti-tumor activity in prostate cancer via inhibition of AR transcriptional activity. This has been proposed to occur by various mechanisms including taxane-mediated induction of AR transcriptional corepressors and prevention of microtubule-mediated transit of AR to the nucleus. ${ }^{92,96,97}$

These data suggest the prostate-specific component of taxane efficacy may be lost in tumors that have developed resistance to AR pathway inhibition. Consistent with this hypothesis, a study reported in abstract form found that metastatic CRPC patients who had early development of castration resistance ( $<12$ months) had a shorter TTP and shorter OS compared to patients with more prolonged sensitivity to androgen axis suppression. ${ }^{98}$

A published analysis of the efficacy of docetaxel in patients who had progressed on the Phase I/II studies of abiraterone showed $>50 \%$ PSA declines in only $26 \%$ of patients compared to $45 \%$ in the TAX 327 study. ${ }^{99}$ Moreover, all patients who had failed to achieve a PSA response on abiraterone were also refractory to docetaxel. A second study reported in abstract form found median OS from the first dose of docetaxel to be 65 months in patients treated with abiraterone or enzalutamide after salvage cabazitaxel therapy, but only 39 months in those receiving these agents after docetaxel but before cabazitaxel. ${ }^{100}$ At present these observations remain hypothesis generating.

\section{Mechanisms of resistance to abiraterone}

Although clinical responses to abiraterone have been impressive, not all men respond, the duration of response is variable, and a majority eventually progress with a rising PSA. While the mechanisms determining resistance to abiraterone have not been fully elucidated, emerging data from clinical and preclinical studies suggest several possibilities.

Clinical data demonstrate that abiraterone effectively suppresses serum androgens. ${ }^{63,64}$ In addition, higher levels of 
AR and CYP17A staining in pretreatment tumor-infiltrated bone marrow biopsies from men with CRPC were associated with longer responses to abiraterone treatment, supporting CYP17A mediated androgen production as the target of abiraterone activity. ${ }^{41}$ However, the efficacy of abiraterone in suppressing tumor androgens in men with CRPC remains to be demonstrated. In this regard, preclinical studies have provided the first in vivo evidence that the primary mechanism of action of abiraterone is via suppression of tumor androgen levels. Treatment of CRPC xenograft models with abiraterone significantly inhibited tumor growth, serum PSA, and intratumoral androgen levels. ${ }^{101,102}$ While androgen levels remained suppressed in some tumors recurring after therapy, other tumors demonstrated increasing levels of T and DHT.

Importantly, multiple mechanisms directed at maintaining AR signaling were observed in the abiraterone-treated tumors. These included upregulated expression of full-length $\mathrm{AR}$ and ligand-independent $\mathrm{AR}$ variants, as well as induction of steroidogenic genes (including the target gene, CYP17A), several of which showed strong correlations with DHT levels in recurrent tumors. ${ }^{101,102}$ Clinically, development of resistance to abiraterone has not been associated with an increase in serum androgen levels or in bone marrow aspirate $T$ levels (which contrasts with the increase in circulating adrenal androgens that is seen in men progressing on ketoconazole). ${ }^{71}$ However, numerous studies have shown that serum is not a good indicator of tumor androgen levels. ${ }^{6,103}$

\section{Abiraterone withdrawal syndrome}

Other potential mechanisms of resistance include activation of mutant AR by noncanonical ligands. Interestingly, recent case reports describe instances of an "abiraterone withdrawal syndrome", in which (generally transient) PSA declines occur following discontinuation of abiraterone. ${ }^{104-106}$ To date, abiraterone has not been found to elicit AR agonist activity (as is seen following treatment with AR antagonists such as bicalutamide, flutamide, and now enzalutamide), ${ }^{37,107}$ perhaps because it is a relatively weak AR antagonist. ${ }^{57} \mathrm{An}$ alternative explanation is the development of AR mutations which allow AR activation by exogenous corticosteroids or steroid precursors upstream of CYP17A.

In particular, inhibition of CYP17A is associated with a rise in circulating levels of upstream progesterone precursors, ${ }^{63,64}$ which have been shown to activate AR bearing certain mutations. ${ }^{31,57}$ Notably, a Phase II study of singleagent abiraterone found that initiation of dexamethasone at progression (to decrease ACTH-driven stimulation of steroid precursors) reversed resistance in $33 \%$ of evaluable patients (although this could also reflect an intrinsic anti-tumor activity of dexamethasone). ${ }^{72}$ In contrast, however, the exogenous glucocorticoids or mineralocorticoid antagonists used to ameliorate the side effects of abiraterone may themselves be able to activate mutant AR. ${ }^{35}$ Alternatively, signaling via the glucocorticoid receptor has been shown to activate AR-regulated genes in the absence of ligand, ${ }^{108}$ suggesting another route by which discontinuation of therapy could lead to a withdrawal response.

While further study is clearly needed, these findings are collectively consistent with the clinical observation that patients progress on abiraterone with a rising PSA, strongly suggesting that the AR axis remains a critical target in abiraterone-resistant tumors.

\section{Conclusion and future directions}

Many important questions regarding the use of abiraterone remain to be answered including optimal dosing strategies, its role in different disease settings (eg, castration sensitive, biochemically recurrent, or localized disease), and - in all disease settings - whether abiraterone in sequential or combinatorial treatment strategies will yield the most durable responses.

Both clinical and preclinical data suggest abirateroneresistance is associated with reactivation of AR signaling. That the AR and a second enzyme involved in androgen synthesis ( $3 \beta$-hydroxysteroid dehydrogenase) are inhibited by higher concentrations of abiraterone suggests dose escalation may be a viable strategy to target AR-related mechanisms of abiraterone resistance. This concept is currently under evaluation in two studies of men with metastatic CRPC (NCT01503229, NCT01637402). ${ }^{109,110}$

The persistent AR activation in abiraterone-resistant tumors also provides a compelling rationale for combining abiraterone with potent AR inhibitors such as enzalutamide or ARN-509 rather than sequential treatment strategies which may allow alternative pathways of AR activation to emerge. Although historic studies of combined androgen blockade have yielded small gains in OS versus ADT alone, the markedly more potent drug combinations now available hold significant promise for increasing the efficacy of multi-targeted AR pathway blockade. Studies evaluating the combination of abiraterone and enzalutamide or ARN-509 in men with metastatic CRPC (NCT01650194, NCT01949337, NCT01792687) $)^{111-113}$ as well as in men with localized disease prior to prostatectomy (NCT01946165) ${ }^{114}$ are planned or ongoing.

Early use of abiraterone or potent combined AR blockade may be particularly effective in hormone-naïve tumors which 
have not yet had the opportunity to develop resistance. The Systemic Therapy in Advancing or Metastatic Prostate Cancer: Evaluation of Drug Efficacy (STAMPEDE) study is an ongoing trial comparing ADT with and without abiraterone in high-risk patients with biochemical recurrence or newly diagnosed metastatic patients (NCT00268476), ${ }^{115}$ as are several others (NCT01715285). ${ }^{116}$ Other studies are evaluating the efficacy of abiraterone and ADT in combination with salvage radiotherapy for biochemical recurrence following prostatectomy (NCT01780220). ${ }^{117}$

Similarly, the efficacy of abiraterone is being explored in combination with prostatectomy or radiotherapy in men with localized disease (NCT01023061, NCT01717053, NCT01751451). ${ }^{118-120}$ In this regard, neoadjuvant studies of multi-targeted AR blockade using LH-releasing hormone agonists combined with bicalutamide, dutasteride, and ketoconazole or LH-releasing hormone agonists combined with abiraterone have demonstrated higher pathologic response rates than previously observed in historic studies of ADT prior to prostatectomy. ${ }^{121,122}$

While the limited number of studies reported to date have identified AR-related mechanisms of resistance to abiraterone, it is likely that other signaling and survival pathways will also be found to play important roles in determining response and resistance. As such, numerous studies evaluating the combination of abiraterone with cytotoxic chemotherapy and targeted agents such as dasatinib (Src inhibitor), veliparib (PARP inhibitor), cabozantinib (c-Met and VEGFR2 inhibitor), alisertib (aurora kinase inhibitor), OGX-427 (HSP27 inhibitor), AT13387 (HSP90 inhibitor), BKM120 (PI3K inhibitor), BEZ235 (dual PI3K/mTOR inhibitor), and ABT264 (Bcl-2 inhibitor) are planned or underway.

In conclusion, numerous studies evaluating the sequencing and combination of abiraterone in multiple disease settings are underway. Rapid accrual and completion of these studies will be imperative for determining rational treatment strategies with the highest likelihood of durable efficacy. Furthermore, the molecular heterogeneity that characterizes CRPC tumors combined with the growing number of oncogenic drivers for which targeted agents are being developed highlights the critical need for embedding correlative studies within these studies and pursuing the development of predictive biomarkers.

\section{Acknowledgments}

Damon Runyon Cancer Research Foundation (Damon Runyon-Genentech Clinical Investigator Award CI-40-08); National Institutes of Health (Pacific Northwest Prostate Cancer SPORE P50 CA97186); Department of Defense
Congressionally Directed Medical Research Program; and Prostate Cancer Foundation.

\section{Disclosure}

Elahe A Mostaghel has received honoraria from Janssen.

\section{References}

1. Scher HI, Sawyers CL. Biology of progressive, castration-resistant prostate cancer: directed therapies targeting the androgen-receptor signaling axis. J Clin Oncol. 2005;23(32):8253-8261.

2. Geller J, Liu J, Albert J, Fay W, Berry CC, Weis P. Relationship between human prostatic epithelial cell protein synthesis and tissue dihydrotestosterone level. Clin Endocrinol (Oxf). 1987;26(2):155-161.

3. Mohler JL, Gregory CW, Ford $\mathrm{OH} 3 \mathrm{rd}$, et al. The androgen axis in recurrent prostate cancer. Clin Cancer Res. 2004;10(2):440-448.

4. Nishiyama T, Hashimoto Y, Takahashi K. The influence of androgen deprivation therapy on dihydrotestosterone levels in the prostatic tissue of patients with prostate cancer. Clin Cancer Res. 2004;10(21): 7121-7126.

5. Page ST, Lin DW, Mostaghel EA, et al. Persistent intraprostatic androgen concentrations after medical castration in healthy men. JClin Endocrinol Metab. 2006;91(10):3850-3856.

6. Montgomery RB, Mostaghel EA, Vessella R, et al. Maintenance of intratumoral androgens in metastatic prostate cancer: a mechanism for castration-resistant tumor growth. Cancer Res. 2008;68(11):4447-4454.

7. Culig Z, Hoffmann J, Erdel M, et al. Switch from antagonist to agonist of the androgen receptor bicalutamide is associated with prostate tumour progression in a new model system. Br J Cancer. 1999;81(2): $242-251$.

8. Gregory CW, Johnson RT Jr, Mohler JL, French FS, Wilson EM. Androgen receptor stabilization in recurrent prostate cancer is associated with hypersensitivity to low androgen. Cancer Res. 2001;61(7): 2892-2898.

9. Gregory CW, Hamil KG, Kim D, et al. Androgen receptor expression in androgen-independent prostate cancer is associated with increased expression of androgen-regulated genes. Cancer Res. 1998;58(24): 5718-5724.

10. Mohler JL, Morris TL, Ford OH 3rd, Alvey RF, Sakamoto C, Gregory CW. Identification of differentially expressed genes associated with androgen-independent growth of prostate cancer. Prostate. 2002;51(4):247-255.

11. Scher HI, Buchanan G, Gerald W, Butler LM, Tilley WD. Targeting the androgen receptor: improving outcomes for castration-resistant prostate cancer. Endocr Relat Cancer. 2004;11(3):459-476.

12. Labrie F, Belanger A, Simard J, Luu-The V, Labrie C. DHEA and peripheral androgen and estrogen formation: intracinology. Ann NY Acad Sci. 1995;774:16-28.

13. Locke JA, Wasan KM, Nelson CC, Guns ES, Leon CG. Androgenmediated cholesterol metabolism in LNCaP and PC-3 cell lines is regulated through two different isoforms of acyl-coenzyme A: cholesterol acyltransferase (ACAT). Prostate. 2008;68(1):20-33.

14. Locke JA, Guns ES, Lubik AA, et al. Androgen levels increase by intratumoral de novo steroidogenesis during progression of castrationresistant prostate cancer. Cancer Res. 2008;68(15):6407-6415.

15. Leon CG, Locke JA, Adomat HH, et al. Alterations in cholesterol regulation contribute to the production of intratumoral androgens during progression to castration-resistant prostate cancer in a mouse xenograft model. Prostate. 2010;70(4):390-400.

16. Mostaghel EA, Solomon KR, Pelton K, Freeman MR, Montgomery RB. Impact of circulating cholesterol levels on growth and intratumoral androgen concentration of prostate tumors. PLoS One. 2012;7(1):e30062.

17. Stanbrough M, Bubley GJ, Ross K, et al. Increased expression of genes converting adrenal androgens to testosterone in androgen-independent prostate cancer. Cancer Res. 2006;66(5):2815-2825. 
18. Hofland J, van Weerden WM, Dits NF, et al. Evidence of limited contributions for intratumoral steroidogenesis in prostate cancer. Cancer Res. 2010;70(3):1256-1264.

19. Bubendorf L, Kononen J, Koivisto P, et al. Survey of gene amplifications during prostate cancer progression by high-throughout fluorescence in situ hybridization on tissue microarrays. Cancer Res. 1999;59(4): 803-806.

20. Ford OH 3rd, Gregory CW, Kim D, Smitherman AB, Mohler JL. Androgen receptor gene amplification and protein expression in recurrent prostate cancer. J Urol. 2003;170(5):1817-1821.

21. Linja MJ, Savinainen KJ, Saramaki OR, Tammela TLJ, Vessella RL, Visakorpi T. Amplification and overexpression of androgen receptor gene in hormone-refractory prostate cancer. Cancer Res. 2001;61(9): 3550-3555.

22. Visakorpi T, Hyytinen E, Koivisto P, et al. In vivo amplification of the androgen receptor gene and progression of human prostate cancer. Nat Genet. 1995;9(4):401-406.

23. van der Kwast TH, Schalken J, Ruizeveld de Winter JA, et al. Androgen receptors in endocrine-therapy-resistant human prostate cancer. Int $J$ Cancer. 1991;48(2):189-193.

24. Ruizeveld de Winter JA, Janssen PJ, Sleddens HM, et al. Androgen receptor status in localized and locally progressive hormone refractory human prostate cancer. Am J Pathol. 1994;144(4):735-746.

25. Taplin ME, Bubley GJ, Shuster TD, et al. Mutation of the androgenreceptor gene in metastatic androgen-independent prostate cancer. N Engl J Med. 1995;332(21):1393-1398.

26. Holzbeierlein J, Lal P, LaTulippe E, et al. Gene expression analysis of human prostate carcinoma during hormonal therapy identifies androgenresponsive genes and mechanisms of therapy resistance. Am J Pathol. 2004;164(1):217-227.

27. Latil A, Bieche I, Vidaud D, et al. Evaluation of androgen, estrogen (ER alpha and ER beta), and progesterone receptor expression in human prostate cancer by real-time quantitative reverse transcription-polymerase chain reaction assays. Cancer Res. 2001;61(5):1919-1926.

28. Miyamoto H, Rahman MM, Chang C. Molecular basis for the antiandrogen withdrawal syndrome. J Cell Biochem. 2004;91(1):3-12.

29. Chen CD, Welsbie DS, Tran C, et al. Molecular determinants of resistance to antiandrogen therapy. Nat Med. 2004;10(1):33-39.

30. Brooke GN, Bevan CL. The role of androgen receptor mutations in prostate cancer progression. Curr Genomics. 2009;10(1):18-25.

31. Culig Z, Hobisch A, Cronauer MV, et al. Mutant androgen receptor detected in an advanced-stage prostatic carcinoma is activated by adrenal androgens and progesterone. Mol Endocrinol. 1993;7(12):1541-1550.

32. Tan J, Sharief Y, Hamil KG, et al. Dehydroepiandrosterone activates mutant androgen receptors expressed in the androgen-dependent human prostate cancer xenograft CWR22 and LNCaP cells. Mol Endocrinol. 1997;11(4):450-459.

33. Taplin ME. Drug insight: role of the androgen receptor in the development and progression of prostate cancer. Nat Clin Pract Oncol. 2007;4(4):236-244.

34. Yuan X, Balk SP. Mechanisms mediating androgen receptor reactivation after castration. Urol Oncol. 2009;27(1):36-41.

35. Zhao XY, Malloy PJ, Krishnan AV, et al. Glucocorticoids can promote androgen-independent growth of prostate cancer cells through a mutated androgen receptor. Nat Med. 2000;6(6):703-706.

36. Steinkamp MP, O’Mahony OA, Brogley M, et al. Treatment-dependent androgen receptor mutations in prostate cancer exploit multiple mechanisms to evade therapy. Cancer Res. 2009;69(10):4434-4442.

37. Korpal M, Korn JM, Gao X, et al. An F876L mutation in androgen receptor confers genetic and phenotypic resistance to MDV3100 (enzalutamide). Cancer Discov. 2013;3(9):1030-1043.

38. Balbas MD, Evans MJ, Hosfield DJ, et al. Overcoming mutationbased resistance to antiandrogens with rational drug design. Elife. 2013;2:e00499.

39. Joseph JD, Lu N, Qian J, et al. A clinically relevant androgen receptor mutation confers resistance to second-generation antiandrogens enzalutamide and ARN-509. Cancer Discov. 2013;3(9):1020-1029.
40. Guo Z, Yang X, Sun F, et al. A novel androgen receptor splice variant is up-regulated during prostate cancer progression and promotes androgen depletion-resistant growth. Cancer Res. 2009;69(6):2305-2313.

41. Hu R, Dunn TA, Wei S, et al. Ligand-independent androgen receptor variants derived from splicing of cryptic exons signify hormonerefractory prostate cancer. Cancer Res. 2009;69(1):16-22.

42. Zhao JJ, Lin J, Lwin T, et al. microRNA expression profile and identification of miR-29 as a prognostic marker and pathogenetic factor by targeting CDK6 in mantle cell lymphoma. Blood. 2010;115(13): 2630-2639.

43. Hornberg E, Ylitalo EB, Crnalic S, et al. Expression of androgen receptor splice variants in prostate cancer bone metastases is associated with castration-resistance and short survival. PLoS One. 2011;6(4):e19059.

44. Haile S, Sadar MD. Androgen receptor and its splice variants in prostate cancer. Cell Mol Life Sci. 2011;68(24):3971-3981.

45. Greenberg E. Endocrine therapy in the management of prostatic cancer. Clin Endocrinol Metab. 1980;9(2):369-381.

46. Robinson MR, Shearer RJ, Fergusson JD. Adrenal suppression in the treatment of carcinoma of the prostate. Br J Urol. 1974;46(5): $555-559$.

47. Samson DJ, Seidenfeld J, Schmitt B, et al. Systematic review and metaanalysis of monotherapy compared with combined androgen blockade for patients with advanced prostate carcinoma. Cancer. 2002;95(2): 361-376.

48. Schmitt B, Bennett C, Seidenfeld J, Samson D, Wilt T. Maximal androgen blockade for advanced prostate cancer [review]. Cochrane Database Syst Rev. 2000;2:CD001526.

49. Caubet JF, Tosteson TD, Dong EW, et al. Maximum androgen blockade in advanced prostate cancer: a meta-analysis of published randomized controlled trials using nonsteroidal antiandrogens. Urology. 1997;49(1): 71-78

50. Small EJ, Ryan CJ. The case for secondary hormonal therapies in the chemotherapy age. J Urol. 2006;176(6 Pt 2):S66-S71.

51. de Bono JS, Logothetis CJ, Fizazi K, et al. Abiraterone acetate (AA) plus low dose prednisone (P) improves overall survival (OS) in patients (pts) with metastatic castration-resistant prostate cancer (mCRPC) who have progressed after docetaxel-based chemotherapy (chemo): results of COU-AA-301, a randomized double-blind placebo-controlled Phase III study [abstract]. Ann Oncol. 2010;21(Suppl 8):LBA5.

52. Scher HI, Beer TM, Higano CS, et al. Antitumour activity of MDV3100 in castration-resistant prostate cancer: a Phase I-II study. Lancet. 2010;375(9724):1437-1446.

53. Efstathiou E, Titus M, Tsavachidou D, et al. Effects of abiraterone acetate on androgen signaling in castrate-resistant prostate cancer in bone. J Clin Oncol. 2012;30(6):637-643.

54. Handratta VD, Vasaitis TS, Njar VC, et al. Novel C-17-heteroaryl steroidal CYP17 inhibitors/antiandrogens: synthesis, in vitro biological activity, pharmacokinetics, and antitumor activity in the LAPC4 human prostate cancer xenograft model. J Med Chem. 2005;48(8): 2972-2984.

55. Rowlands MG, Barrie SE, Chan F, et al. Esters of 3-pyridylacetic acid that combine potent inhibition of 17 alpha-hydroxylase/C17,20-lyase (cytochrome P45017 alpha) with resistance to esterase hydrolysis. J Med Chem. 1995;38(21):4191-4197.

56. Attard G, Reid AH, Auchus RJ, et al. Clinical and biochemical consequences of CYP17A1 inhibition with abiraterone given with and without exogenous glucocorticoids in castrate men with advanced prostate cancer. J Clin Endocrinol Metab. 2012;97(2): 507-516.

57. Richards J, Lim AC, Hay CW, et al. Interactions of abiraterone eplerenone, and prednisolone with wild-type and mutant androgen receptor: a rationale for increasing abiraterone exposure or combining with MDV3100. Cancer Res. 2012;72(9):2176-2182.

58. Soifer HS, Souleimanian N, Wu S, et al. Direct regulation of androgen receptor activity by potent CYP17 inhibitors in prostate cancer cells. J Biol Chem. 2012;287(6):3777-3787. 
59. Eil C. Ketoconazole binds to the human androgen receptor. Horm Metab Res. 1992;24(8):367-370.

60. Li R, Evaul K, Sharma KK, et al. Abiraterone inhibits $3 \beta$-hydroxysteroid dehydrogenase: a rationale for increasing drug exposure in castrationresistant prostate cancer. Clin Cancer Res. 2012;18(13):3571-3579.

61. Barrie SE, Potter GA, Goddard PM, Haynes BP, Dowsett M, Jarman M. Pharmacology of novel steroidal inhibitors of cytochrome P450(17) alpha (17 alpha-hydroxylase/C17-20 lyase). J Steroid Biochem Mol Biol. 1994;50(5-6):267-273.

62. O'Donnell A, Judson I, Dowsett M, et al. Hormonal impact of the 17alphahydroxylase/C(17,20)-lyase inhibitor abiraterone acetate (CB7630) in patients with prostate cancer. Br J Cancer. 2004;90(12):2317-2325.

63. Attard G, Reid AH, Yap TA, et al. Phase I clinical trial of a selective inhibitor of CYP17, abiraterone acetate, confirms that castrationresistant prostate cancer commonly remains hormone driven. J Clin Oncol. 2008;26(28):4563-4571.

64. Ryan CJ, Smith MR, Fong L, et al. Phase I clinical trial of the CYP17 inhibitor abiraterone acetate demonstrating clinical activity in patients with castration-resistant prostate cancer who received prior ketoconazole therapy. J Clin Oncol. 2010;28(9):1481-1488.

65. Todd M, Meyers ML, Charnas R, Acharya M, Molina A. Fast and flawed or scientifically sound: the argument for administering oral oncology drugs during fasting. J Clin Oncol. 2012;30(8):888-889.

66. Janssen-Ortho Inc., Canada. A Study to Determine the Short-Term Safety of Continuous Dosing of Abiraterone Acetate and Prednisone in Modified Fasting and Fed States to Patients With Metastatic Castration-Resistant Prostate Cancer. Available from: http://clinicaltrials.gov/show/NCT01424930. NLM identifier: NCT01424930. Accessed December 10, 2013.

67. University of Chicago. Food Effect Study of Abiraterone Acetate for Treatment of Patients With Castration-Resistant Prostate Cancer. Available from: http://clinicaltrials.gov/show/NCT01543776. NLM identifier: NCT01543776. Accessed December 10, 2013.

68. Ratain MJ. Flushing oral oncology drugs down the toilet. J Clin Oncol. 2011;29(30):3958-3959.

69. Marbury T, Stonerock R, Tran N, Gonzalez M. A Phase I single dose open-label reduced/staged pharmacokinetic (PK) and safety study of abiraterone acetate (AA) in men with imapired renal function. Eur $J$ Cancer. 2011;47(Suppl 1):S502.

70. Acharya M, Gonzalez M, Mannens G, et al. A Phase I, open-label, single-dose, mass balance study of 14C-labeled abiraterone acetate in healthy male subjects. Xenobiotica. 2013;43(4):379-389.

71. Ryan CJ, Halabi S, Ou SS, Vogelzang NJ, Kantoff P, Small EJ. Adrenal androgen levels as predictors of outcome in prostate cancer patients treated with ketoconazole plus antiandrogen withdrawal: results from a cancer and leukemia group B study. Clin Cancer Res. 2007;13(7):2030-2037.

72. Attard G, Reid AH, A'Hern R, et al. Selective inhibition of CYP17 with abiraterone acetate is highly active in the treatment of castration-resistant prostate cancer. J Clin Oncol. 2009;27(23):3742-3748.

73. Ryan CJ, Shah S, Efstathiou E, et al. Phase II study of abiraterone acetate in chemotherapy-naïve metastatic castration-resistant prostate cancer displaying bone flare discordant with serologic response. Clin Cancer Res. 2011;17(14):4854-4861.

74. Reid AH, Attard G, Danila DC, et al. Significant and sustained antitumor activity in post-docetaxel, castration-resistant prostate cancer with the CYP17 inhibitor abiraterone acetate. J Clin Oncol. 2010;28(9): 1489-1495.

75. Danila DC, Morris MJ, de Bono JS, et al. Phase II multicenter study of abiraterone acetate plus prednisone therapy in patients with docetaxeltreated castration-resistant prostate cancer. J Clin Oncol. 2010;28(9): 1496-1501.

76. Fizazi K, Scher HI, Molina A, et al. Abiraterone acetate for treatment of metastatic castration-resistant prostate cancer: final overall survival analysis of the COU-AA-301 randomised, double-blind, placebocontrolled Phase III study. Lancet Oncol. 2012;13(10):983-992.

77. Goodman OB, Flaig TW, Molina A, et al. Exploratory analysis of the visceral disease (VD) patient subset in COU-AA-301, a Phase III study of abiraterone acetate (AA) in metastatic castration-resistant prostate cancer (mCRPC) [abstract]. J Clin Oncol. 2013;31(Suppl 6):14.
78. Sternberg CN, Molina A, North S, et al. Effect of abiraterone acetate on fatigue in patients with metastatic castration-resistant prostate cancer after docetaxel chemotherapy. Ann Oncol. 2013;24(4):1017-1025.

79. Logothetis CJ, Basch E, Molina A, et al. Effect of abiraterone acetate and prednisone compared with placebo and prednisone on pain control and skeletal-related events in patients with metastatic castration-resistant prostate cancer: exploratory analysis of data from the COU-AA-301 randomised trial. Lancet Oncol. 2012;13(12):1210-1217.

80. Ryan CJ, Smith MR, de Bono JS, et al. Abiraterone in metastatic prostate cancer without previous chemotherapy. $N$ Engl J Med. 2013;368(2): $138-148$.

81. Rathkopf DE, Smith MR, De Bono JS, et al. Updated interim analysis (IA) of COU-AA-302, a randomized Phase III study of abiraterone acetate (AA) in patients (pts) with metastatic castration-resistant prostate cancer (mCRPC) without prior chemotherapy [abstract]. J Clin Oncol. 2013;31(Suppl 6):5.

82. Ferraldeschi R, Sharifi N, Auchus RJ, Attard G. Molecular pathways: inhibiting steroid biosynthesis in prostate cancer. Clin Cancer Res. 2013;19(13):3353-3359.

83. Pia A, Vignani F, Attard G, et al. Strategies for managing ACTH dependent mineralocorticoid excess induced by abiraterone. Cancer Treat Rev. 2013;39(8):966-973

84. Procopio G, Grassi P, Testa I, et al. Safety of abiraterone acetate in castration-resistant prostate cancer patients with concomitant cardiovascular risk factors. Am J Clin Oncol. Epub September 21, 2013.

85. Tolcher AW, Chi KN, Shore ND, et al. Effect of abiraterone acetate plus prednisone on the QT interval in patients with metastatic castrationresistant prostate cancer. Cancer Chemother Pharmacol. 2012;70(2): 305-313.

86. Chi KN, Tolcher A, Lee P, et al. Effect of abiraterone acetate plus prednisone on the pharmacokinetics of dextromethorphan and theophylline in patients with metastatic castration-resistant prostate cancer. Cancer Chemother Pharmacol. 2013;71(1):237-244.

87. Bertilsson L, Dahl ML, Dalen P, Al-Shurbaji A. Molecular genetics of CYP2D6: clinical relevance with focus on psychotropic drugs. $\mathrm{Br}$ J Clin Pharmacol. 2002;53(2):111-122.

88. Janssen Research and Development, LLC. A Study to Evaluate the Effect of Rifampicin on the Pharmacokinetics of Abiraterone in Healthy Male Participants. Available from: http://clinicaltrials.gov/show/ NCT01655147. NLM identifier: NCT01655147. Accessed December $10,2013$.

89. Janssen Research and Development, LLC. A Study to Assess the Effect of Ketoconazole on the Pharmacokinetics of Abiraterone Following Administration of Abiraterone Acetate Tablets in Healthy Adult Men. Available from: http:/clinicaltrials.gov/show/NCT01588782. NLM identifier: NCT01588782. Accessed December 10, 2013.

90. Dendreon. Concurrent Versus Sequential Treatment With Sipuleucel-T and Abiraterone in Men With Metastatic Castrate Resistant Prostate Cancer (mCRPC). Available from: http://clinicaltrials.gov/show/NCT01487863. NLM identifier: NCT01487863. Accessed December 10, 2013.

91. Fitzpatrick JM, de Wit R. Taxane mechanisms of action: potential implications for treatment sequencing in metastatic castration-resistant prostate cancer. Eur Urol. Epub July 25, 2013.

92. Gan L, Chen S, Wang Y, et al. Inhibition of the androgen receptor as a novel mechanism of taxol chemotherapy in prostate cancer. Cancer Res. 2009;69(21):8386-8394.

93. Loriot Y, Bianchini D, Ileana E, et al. Antitumour activity of abiraterone acetate against metastatic castration-resistant prostate cancer progressing after docetaxel and enzalutamide (MDV3100). Ann Oncol. 2013;24(7):1807-1812.

94. Schrader AJ, Boegemann M, Ohlmann CH, et al. Enzalutamide in castration-resistant prostate cancer patients progressing after docetaxel and abiraterone. Eur Urol. 2014;65(1):30-36.

95. Janssen Pharmaceutica N.V., Belgium. Abiraterone With Different Steroid Regimens for Side Effect Related to Mineralcorticoid Excess Prevention in Prostate Cancer Prior to Chemotherapy. Available from: http://clinicaltrials.gov/show/NCT01867710. NLM identifier: NCT01867710. Accessed December 10, 2013. 
96. Darshan MS, Loftus MS, Thadani-Mulero M, et al. Taxane-induced blockade to nuclear accumulation of the androgen receptor predicts clinical responses in metastatic prostate cancer. Cancer Res. 2011;71(18): 6019-6029.

97. Zhu ML, Horbinski CM, Garzotto M, Qian DZ, Beer TM, Kyprianou N. Tubulin-targeting chemotherapy impairs androgen receptor activity in prostate cancer. Cancer Res. 2010;70(20):7992-8002.

98. Huillard O, Albiges L, Eymard JC, et al. Efficacy of docetaxel chemotherapy in metastatic prostate cancer $(\mathrm{mPC})$ patients $(\mathrm{pts})$ experiencing early castration resistance (CR) [abstract]. J Clin Oncol. 2013;31(Suppl):5075.

99. Mezynski J, Pezaro C, Bianchini D, et al. Antitumour activity of docetaxel following treatment with the CYP17A1 inhibitor abiraterone: clinical evidence for cross-resistance? Ann Oncol. 2012;23(11):2943-2947.

100. Angelergues A, Maillet D, Flechon A, et al. Prognostic factors of survival in patients with metastatic castration resistant prostate cancer (mCRPC) treated with cabazitaxel: sequencing might matter [abstract]. J Clin Oncol. 2013;31(Suppl):5063.

101. Mostaghel EA, Marck BT, Plymate SR, et al. Resistance to CYP17A1 inhibition with abiraterone in castration-resistant prostate cancer: induction of steroidogenesis and androgen receptor splice variants. Clin Cancer Res. 2011;17(18):5913-5925.

102. Cai C, Chen S, Ng P, et al. Intratumoral de novo steroid synthesis activates androgen receptor in castration-resistant prostate cancer and is upregulated by treatment with CYP17A1 inhibitors. Cancer Res. 2011;71(20):6503-6513.

103. Mostaghel EA, Page ST, Lin DW, et al. Intraprostatic androgens and androgen-regulated gene expression persist after testosterone suppression: therapeutic implications for castration-resistant prostate cancer. Cancer Res. 2007;67(10):5033-5041.

104. Caffo O, Palermo A, Veccia A, et al. Biochemical and objective response to abiraterone acetate withdrawal: incidence and clinical relevance of a new scenario for castration-resistant prostate cancer. Urology. 2013;82(5):1090-1093

105. Gauthier H, Bousquet G, Pouessel D, Culine S. Abiraterone acetate withdrawal syndrome: does it exist? Case Rep Oncol. 2012;5(2):385-387.

106. Witjes JA. A case of abiraterone acetate withdrawal. Eur Urol. 2013;64(3):517-518.

107. Labrie F, Dupont A, Belanger A, et al. New approach in the treatment of prostate cancer: complete instead of partial withdrawal of androgens. Prostate. 1983;4(6):579-594.

108. Sahu B, Laakso M, Pihlajamaa P, et al. FoxA1 specifies unique androgen and glucocorticoid receptor binding events in prostate cancer cells. Cancer Res. 2013;73(5):1570-1580.

109. University of Washington. Abiraterone Acetate in Treating Patients With Metastatic Hormone-Resistant Prostate Cancer. Available from: http://clinicaltrials.gov/show/NCT01508234. NLM identifier: NCT01503229. Accessed December 10, 2013.

110. Terence Friedlander, MD. A Phase II Study of Increased-Dose Abiraterone Acetate in Patients With Castration Resistant Prostate Cancer. Available from: http://clinicaltrials.gov/show/NCT01637402. NLM identifier: NCT01637402. Accessed December 10, 2013.

111. Astellas Pharma Global Development, Inc. A Study to Determine Safety and Tolerability of Enzalutamide (MDV3100) in Combination With Abiraterone Acetate in Bone Metastatic Castration-Resistant Prostate Cancer Patients. Available from: http://clinicaltrials.gov/show/NCT01650194. NLM identifier: NCT01650194. Accessed December 10, 2013.
112. Alliance for Clinical Trials in Oncology. Enzalutamide With or Without Abiraterone Acetate and Prednisone in Treating Patients With Castration-Resistant Metastatic Prostate Cancer. Available from: http:/clinicaltrials.gov/show/NCT01949337. NLM identifier: NCT01949337. Accessed December 10, 2013.

113. Aragon Pharmaceuticals, Inc. Safety, Tolerability, Pharmacokinetics, and Preliminary Anti-tumor Activity of Ascending Doses of ARN 509 in Combination With Abiraterone Acetate. Available from: http://clinicaltrials.gov/show/NCT01792687. NLM identifier: NCT01792687. Accessed December 10, 2013.

114. M.D. Anderson Cancer Center.Abiraterone Acetate Plus LHRH Agonist and Abiraterone Acetate Plus LHRH Agonist and Enzalutamide. Available from: http://clinicaltrials.gov/show/NCT01946165. NLM identifier: NCT01946165. Accessed December 10, 2013.

115. Medical Research Council.STAMPEDE: Systemic Therapy in Advancing or Metastatic Prostate Cancer: Evaluation of Drug Efficacy: A Multi-Stage Multi-Arm Randomised Controlled Trial. Available from: http://clinicaltrials.gov/show/NCT00268476. NLM identifier: NCT00268476. Accessed December 10, 2013

116. Janssen Research \& Development, LLC. A Study of Abiraterone Acetate Plus Low-Dose Prednisone Plus Androgen Deprivation Therapy (ADT) Versus ADT Alone in Newly Diagnosed Patients With High-Risk, Metastatic Hormone-Naive Prostate Cancer (mHNPC). Available from: http://clinicaltrials.gov/show/NCT01715285. NLM identifier: NCT01715285. Accessed December 10, 2013.

117. UNICANCER. Safety and Efficacy of Radiotherapy Combined With a 6-month LH-RH Agonist and Abiraterone Hormone Therapy Treatment in Biochemically-relapsing Prostate Cancer Following Surgery (CARLHA). Available from: http://clinicaltrials.gov/show/ NCT01780220. NLM identifier: NCT01780220. Accessed December 10,2013

118. University of Washington. Abiraterone Acetate, Prednisone, and Leuprolide Acetate or Goserelin Before and During Radiation Therapy in Treating Patients With Localized or Locally Advanced Prostate Cancer. Available from: http:/clinicaltrials.gov/show/NCT01023061. NLM identifier: NCT01023061. Accessed December 10, 2013.

119. Duke University.Abiraterone, Radiotherapy and Short-Term Androgen Deprivation in Unfavorable Localized Prostate Cancer. Available from: http://clinicaltrials.gov/show/NCT01717053. NLM identifier: NCT01717053. Accessed December 10, 2013.

120. Memorial Sloan-Kettering Cancer Center.3-arm Study of Abiraterone Acetate Alone, Abiraterone Acetate Plus Degarelix, a GnRH Antagonist, and Degarelix Alone for Patients With Prostate Cancer With a Rising PSA or a Rising PSA and Nodal Disease Following Definitive Radical Prostatectomy. Available from: http://clinicaltrials.gov/show/ NCT01751451. NLM identifier: NCT01751451. Accessed December 10,2013

121. Mostaghel EA, Nelson PS, Lange P, et al. Targeted androgen pathway suppression in localized prostate cancer: a randomized clinical trial J Clin Oncol. Epub 2013 Dec 9.

122. Taplin ME, Montgomery RB, Logothetis C, et al. Effect of neoadjuvant abiraterone acetate (AA) plus leuprolide acetate (LHRHa) on PSA, pathological complete response (pCR), and near pCR in localized high-risk prostate cancer (LHRPC): results of a randomized Phase II study [abstract]. J Clin Oncol. 2012;30(Supp1):4521.
Cancer Management and Research

\section{Publish your work in this journal}

Cancer Management and Research is an international, peer-reviewed open access journal focusing on cancer research and the optimal use of preventative and integrated treatment interventions to achieve improved outcomes, enhanced survival and quality of life for the cancer patient The journal welcomes original research, clinical \& epidemiological

\section{Dovepress}

studies, reviews \& evaluations, guidelines, expert opinion \& commentary, case reports \& extended reports. The manuscript management system is completely online and includes a very quick and fair peerreview system, which is all easy to use. Visit http://www.dovepress.com/ testimonials.php to read real quotes from published authors. 